\title{
Correlation functions of one-dimensional Bose-Fermi mixtures
}

\author{
Holger Frahm and Guillaume Palacios \\ Institut für Theoretische Physik, Universität Hannover, Appelstraße 2, 30167 Hannover, Germany
}

(Received 14 July 2005; published 16 December 2005)

\begin{abstract}
We calculate the asymptotic behavior of correlators as a function of the microscopic parameters for an integrable Bose-Fermi mixture with repulsive interaction in one dimension. For two cases, namely polarized and unpolarized fermions the singularities of the momentum distribution functions are characterized as a function of the coupling constant and the relative density of bosons.
\end{abstract}

DOI: 10.1103/PhysRevA.72.061604

PACS number(s): 03.75.Mn, 05.70.Jk, 71.10.Pm

In the past years, the advances in cooling and trapping of atomic gases have opened the possibility to realize quasione-dimensional (1D) systems with tunable strength of the interactions in optical lattices. This gives rise to opportunities for the investigation of the striking phenomena appearing in correlated systems as a consequence of the enhanced quantum fluctuations in reduced spatial dimensions. The observable signatures of these phenomena are encoded in the correlation functions of the system such as the momentum distribution function which can be measured directly in timeof-flight experiments or using Bragg spectroscopy [1]. A setup for the measurement of various density correlation functions has been proposed for the identification of dominant correlations in the atomic gas [2]. Theoretical studies of correlation functions in cold atomic gases have been performed both using analytical methods, e.g., bosonization [3] combined with exact results from integrable models such as the Bose gas with repulsive $\delta$-function interaction [4], and numerically. Additional correlation effects appear when the particles considered have internal degrees of freedom. In cold gases containing different constituent atoms Bose-Fermi mixtures can be realized [5]. Extensive theoretical results exist for 1D Fermi gases due to their equivalence with the Tomonaga-Luttinger (TL) liquids realized by correlated electrons in 1D lattices $[3,6]$. Only recently, theoretical investigations have been extended to Bose-Fermi mixtures: some correlation functions have been calculated numerically in the strong coupling limit $[7,8]$ where the problem simplifies due to the factorization of the many-particle wave function (see, e.g., [9]). For analytical results on these systems one has to go beyond mean-field approximations and use methods which can capture the strong quantum fluctuations in 1D systems. The phase diagram and certain correlation functions of atomic mixtures have been studied in the Luttinger liquid picture $[10,11]$. Without further input, however, these results are limited to the weakly interacting regime since the TL parameters which determine the low-energy theory cannot easily be related to the microscopic parameters describing the underlying gas. Therefore, instabilities predicted within this approach may not appear in a specific realization $[7,12,13]$.

In this paper we establish the relation between the TL and the microscopic parameters for an integrable Bose-Fermi mixture [12]. We employ methods from conformal quantum field theory (CFT) to determine the asymptotic (longdistance, low-energy) behavior of correlation functions in the model from a finite size scaling analysis of the exact spectrum obtained by means of the Bethe ansatz. This approach gives the complete set of critical exponents of the model as a function of the parameters in the microscopic Hamiltonian (see, e.g., $[6,14,15]$ for applications to $1 \mathrm{D}$ correlated electrons). As an application we compute the momentum distribution function of bosons and fermions in the atomic mixture as a function of their respective densities and the effective coupling constant. It should be emphasized that our results can be expected to describe the generic (universal) lowenergy behavior of atomic mixtures. Additional interactions - as long as they do not lead to a phase transition-will merely change the anomalous exponents but not the qualitative behavior of the correlation functions.

The 1D Bose-Fermi mixture of $N=M_{f}+M_{b}$ particles with repulsive interaction $(c>0)$ on a line of length $L$ subject to periodic boundary conditions is described by the integrable Hamiltonian [12]

$$
\mathcal{H}=-\sum_{i=1}^{N} \frac{\partial^{2}}{\partial x_{i}^{2}}+2 c \sum_{i<k} \delta\left(x_{i}-x_{k}\right) .
$$

Here $M_{f}=M_{\uparrow}+M_{\downarrow}$ of the particles are fermions carrying spin $\sigma=\uparrow, \downarrow$ and $M_{b}$ of them are bosons. Note that fixing the particle numbers breaks the apparent symmetry of the Hamiltonian arising from the equality of the particles' masses and their mutual interactions. The many-particle eigenstates of (1) are parametrized by the solutions of the Bethe ansatz equations (BAE) [12]

$$
\begin{gathered}
\exp \left(i q_{j}^{(0)} L\right)=\prod_{k=1}^{M_{1}} e_{c}\left(q_{j}^{(0)}-q_{k}^{(1)}\right) \\
\prod_{j=1}^{M_{0}} e_{c}\left(q_{k}^{(1)}-q_{j}^{(0)}\right) \prod_{\ell=1}^{M_{2}} e_{c}\left(q_{k}^{(1)}-q_{\ell}^{(2)}\right)=\prod_{k^{\prime} \neq k}^{M_{1}} e_{2 c}\left(q_{k}^{(1)}-q_{k^{\prime}}^{(1)}\right), \\
\prod_{k=1}^{M_{1}} e_{c}\left(q_{\ell}^{(2)}-q_{k}^{(1)}\right)=1
\end{gathered}
$$

where $e_{a}(x)=(x+i a / 2) /(x-i a / 2)$ and $M_{0}=N, M_{1}=N-M_{\uparrow}$, $M_{2}=M_{b}$. The corresponding eigenvalue of (1) is $E$ $=\sum_{j=1}^{N}\left(q_{j}^{(0)}\right)^{2}$. In the thermodynamic limit $L \rightarrow \infty$ with $M_{i} / L$ kept fixed, the root configurations $\left\{q^{(i)}\right\}$ of Eqs. (2) can be 
described by distribution functions $\rho_{i}$ which, as a consequence of (2) are solutions to [12]

$$
\rho_{i}(x)=\rho_{i}^{(0)}+\sum_{j}\left(\hat{K}_{i j} \otimes \rho_{j}\right)(x) .
$$

Here $\rho_{i}^{(0)}=(c / 2 \pi) \delta_{i 0}$ and $\hat{K}_{i j}$ are linear integral operators acting as $\left(\int_{j} \equiv \int_{-Q_{j}}^{Q_{j}} \mathrm{~d} y\right)$

$$
\left(\hat{K}_{i j} \otimes f\right)(x)=\int_{j} k_{i j}(x-y) f(y) .
$$

The kernels of these integral operators are $k_{i j}(x)$ $=a_{1}(x) \delta_{|i-j|, 1}-a_{2}(x) \delta_{i, 1} \delta_{j, 1} \quad$ where $2 \pi a_{n}(x)=4 n /\left(4 x^{2}+n^{2}\right)$. The properties of the system are completely characterized by the densities $m_{i} \equiv M_{i} / L=\int_{i} \rho^{(i)}(x)$ of the components of the mixture (these relations determine the boundaries $Q_{i}$ of the above integral equations) and the dimensionless coupling strength $\gamma=L c / N$. For later use we also introduce the fraction of bosons in the system $\alpha=M_{b} / N$.

Generically, i.e., for $m_{i}>0$, there are three modes of collective elementary excitations above the many-particle ground state of (1). Their dispersion $\epsilon_{i}(k)$ is linear at low energies with different sound velocities $v_{i}=\partial \epsilon_{i} / \partial k, i=0,1,2$ [13]. These quantities determine the finite size scaling behavior of the ground-state energy

$$
E_{0}-L \epsilon_{\infty}=-\frac{\pi}{6 L} \sum_{i} v_{i}+o\left(\frac{1}{L}\right) .
$$

Physical excitations of the system are combinations of the elementary ones. Due to the interacting nature of the system the different modes are coupled and excitations in one of the modes shift the energies in the other ones. In general, this effect can be described in terms of generalized susceptibilities which may be determined in an experiment or numerically from studies of small systems [6]. For the Bethe ansatz solvable models it is possible to describe the coupling of the modes in terms of the dressed charge matrix [16] which in this case reads

$$
Z_{i j}=\xi_{i j}\left(Q_{i}\right) .
$$

The functions $\xi_{i j}$ are given in terms of integral equations $\xi_{i j}(x)=\delta_{i j}+\Sigma_{k}\left(\hat{K}_{i k} \otimes \xi_{k j}\right)(x)$.

$Z$ determines the general form of the finite size corrections to the energies of low-lying excitations

$$
\begin{aligned}
\Delta E(\Delta \mathbf{M}, \mathbf{D})= & \frac{2 \pi}{L}\left(\frac{1}{4} \Delta \mathbf{M}^{\top}\left(Z^{\top}\right)^{-1} V Z^{-1} \Delta \mathbf{M}+\mathbf{D}^{\top} Z V Z^{\top} \mathbf{D}\right. \\
& \left.+\sum_{k} v_{k}\left(N_{k}^{+}+N_{k}^{-}\right)\right)+o\left(\frac{1}{L}\right) .
\end{aligned}
$$

Here, $V=\operatorname{diag}\left(v_{0}, v_{1}, v_{2}\right)$ is a $3 \times 3$ matrix of the sound velocities, $N_{k}^{ \pm}$are nonnegative integers, $\Delta M$ is a vector of integers denoting the change of $M_{i}$ with respect to the ground state for charged excitations. The $D_{i}$ are integers or half-odd integers according to

$$
D_{0} \sim\left(\Delta M_{0}+\Delta M_{1}\right) / 2=\Delta M_{\uparrow} / 2 \bmod 1,
$$

$$
\begin{aligned}
& D_{1} \sim\left(\Delta M_{0}+\Delta M_{2}\right) / 2=\Delta M_{f} / 2 \bmod 1, \\
& D_{2} \sim\left(\Delta M_{1}+\Delta M_{2}\right) / 2=\Delta M_{\downarrow} / 2 \bmod 1,
\end{aligned}
$$

and enumerate finite momentum transfer processes,

$$
\begin{aligned}
\Delta P(\Delta \mathbf{M}, \mathbf{D})= & \frac{2 \pi}{L}\left(\Delta \mathbf{M}^{\top} \cdot \mathbf{D}+\sum_{k}\left(N_{k}^{+}-N_{k}^{-}\right)\right)+2 k_{F, \uparrow} D_{0} \\
& +2 k_{F, \downarrow}\left(D_{0}+D_{1}\right)+2 k_{B} \sum_{j} D_{j} .
\end{aligned}
$$

Here $k_{F, \sigma}=\pi M_{\sigma} / L$ are the Fermi momenta of the fermion components, $k_{B}=\pi M_{b} / L$ is the corresponding quantity for the interacting bosons.

In the framework of CFT [17] the finite size spectrum (5) and (7) can be understood as that of a critical theory based on the product of three Virasoro algebras each having central charge $C=1[6,14]$. Correlation functions of a general operator in the theory-characterized by the quantum numbers $\Delta M_{i}$ and $D_{i}$-will contain contributions from these three sectors. The simplest ones, analogues of primary fields in the CFT, have correlation functions (in Euclidean time $\tau$ )

$$
\begin{aligned}
& \left\langle\phi_{\Delta}(x, \tau) \phi_{\Delta}(0,0)\right\rangle \\
& =\frac{\exp \left[2 i D_{0} k_{F, \uparrow} x+2 i\left(D_{0}+D_{1}\right) k_{F, \downarrow} x+2 i\left(\sum_{j} D_{j}\right) k_{B} x\right]}{\prod_{k}\left(v_{k} \tau+i x\right)^{2 \Delta_{k}^{+}}\left(v_{k} \tau-i x\right)^{2 \Delta_{k}^{-}}} .
\end{aligned}
$$

The operators $\phi_{\Delta}$ are characterized by their scaling dimensions $\Delta_{k}^{ \pm}$in the chiral (left and right moving) components of all three constituent theories. The latter are uniquely determined from the finite size energies (7) and momenta (9) and form towers starting at

$$
2 \Delta_{k}^{ \pm}=\left(\sum_{j} Z_{k j} D_{j} \pm \frac{1}{2} \sum_{j} \Delta M_{j}\left(Z^{-1}\right)_{j k}\right)^{2} .
$$

The asymptotic exponential decay of correlation functions in a large but finite system or at finite temperature $T$ can be obtained from (10) by conformal invariance. For example, at $T>0$ the denominators in (10) have to be replaced by $\left(v_{k} \tau \pm i x\right)^{-2 \Delta_{k}^{ \pm}} \rightarrow\left[\pi T / v_{k} \sin \pi T\left(\tau \pm i x / v_{k}\right)\right]^{2 \Delta_{k}^{ \pm}}$.

With (11) the critical exponents which determine the long-distance asymptotics of any correlation function are known as soon as we have computed the dressed charge matrix (6). To calculate the correlation functions of a given local operator $\mathcal{O}$ in the microscopic theory (1) one needs to know its expansion in terms of the fields $\phi_{\Delta}$ of the CFT. Usually, this expansion is not known but $\mathcal{O}$ and $\phi_{\Delta}$ have to generate the same set of selection rules in calculating the correlation function. This drastically reduces the number of possible terms in the expansion: As an example consider the bosonic Green's function $G_{b}(x, \tau)=\left\langle\Psi_{b}(x, \tau) \Psi_{b}^{\dagger}(0,0)\right\rangle$ : clearly $\Psi_{b}^{\dagger}$ generates a state with $\Delta M_{b}=1$ which implies $\Delta M_{j} \equiv 1$ in (11). By (8) the quantum numbers $D_{j}$ are further restricted to integers: the uniform part of the $G_{b}(x)$ $\sim|x|^{-1 / 2 K_{b}}$ is described by the operator with $D_{j} \equiv 0$ which allows to identify the TL parameter $K_{b}[18]$ from (11). The 
interactions lead to additional contributions to $G_{b}$ oscillating with wave numbers $k_{0}=2 k_{F \sigma}, 2 k_{B}, \ldots$.

For a comparison with experimental data one is often interested in Fourier transforms of the two-point correlation functions given above. The large distance behavior of (10) determines the singularities of spectral functions near $\omega$ $\approx \pm v_{k}\left(k-k_{0}\right)$ (see, e.g., [6]). Quantities accessible in experiments with cold gases [1] are the momentum distribution functions of the constituent particles. For the bosons this is the Fourier transform of the equal time Green's function $G_{b}(x)$. From (10) its singularities at wave numbers $k_{0}$ are then $n_{b}(k) \sim\left|k-k_{0}\right|^{\nu_{b}}$ near $k \approx k_{0}$. The exponent $\nu_{b}$ is the minimal value of $2 \Sigma_{k}\left(\Delta_{k}^{+}+\Delta_{k}^{-}\right)-1$ compatible with the quantum numbers $\Delta M$ and the selection rules for the $D$ for the given $k_{0}$, e.g., $1 / 2 K_{b}=\nu_{b}+1=1 / 4 \Sigma_{k}\left[\Sigma_{j}\left(Z_{j k}^{-1}\right)\right]^{2}$ for $k_{0}=0$.

Using the same procedure for the fermionic Green's function $G_{\sigma}(x, \tau)=\left\langle\Psi_{\sigma}(x, \tau) \Psi_{\sigma}^{\dagger}(0,0)\right\rangle$ we find that their asymptotic behavior is determined by the conformal fields with $\Delta M_{0}=1, \Delta M_{1}=\Delta M_{2}=0$, half-odd integers $D_{0}, D_{1}$, and integer $D_{2}$ for $G_{\uparrow}$ and $\Delta M_{0}=1=\Delta M_{1}, \Delta M_{2}=0$, half-odd integers $D_{1}, D_{2}$, and integer $D_{0}$ for $G_{\downarrow}$. Again, the singularities of the fermions' distribution functions $n_{\sigma}(k)$ follow from (10). Near $k_{0}-k_{F \sigma}=0, \pm 2 k_{B}, \ldots$ they are given by

$$
n_{\sigma}(k) \sim \operatorname{sgn}\left(k-k_{0}\right)\left|k-k_{0}\right|^{\nu_{f}} \text { for } k \approx k_{0} .
$$

$\nu_{f}$ is related to the dimensions (11) for the quantum numbers $\Delta M$ and $D$ as $\nu_{b}$ above. The Fermi distribution of noninteracting particles corresponds to $\nu_{f}\left(k_{F \sigma}\right)=0$.

In the following we consider two cases of particular relevance [11-13], namely (i) the unpolarized case where $M_{\uparrow}$ $=M_{\downarrow}=M_{f} / 2$ and the ground state of the system is invariant under rotations in the spin index of the fermions and (ii) the fully polarized case where there is only one spin component of the fermions.

The unpolarized gas. For $Q_{1}=\infty$ one obtains $M_{\uparrow}=M_{\downarrow}$ from (3), i.e., with vanishing net magnetization. In this case the dressed charge matrix (6) takes the form

$$
Z=\frac{1}{2}\left(\begin{array}{ccc}
2 \zeta_{00} & \left(\zeta_{00}+\zeta_{01}\right) & 2 \zeta_{01} \\
0 & \sqrt{2} & 0 \\
2 \zeta_{10} & \left(\zeta_{10}+\zeta_{11}\right) & 2 \zeta_{11}
\end{array}\right)
$$

Here the Wiener-Hopf method has been used to determine $Z_{11}=1 / \sqrt{2}$ and $\zeta_{0 j}=\zeta_{0 j}\left(Q_{0}\right), \zeta_{1 j}=\zeta_{1 j}\left(Q_{2}\right)$. The functions $\zeta_{i j}(x)$ are given by

$$
\zeta_{i j}(x)=\delta_{i j}+\int_{0} R(x-y) \zeta_{0 j}(y)+\int_{2} R(x-y) \zeta_{1 j}(y)
$$

with $R(x)=(1 / \pi) \int_{0}^{\infty} d \omega e^{-|\omega| / 2} \cos (\omega x) / \cosh (\omega / 2)$. Using Eq. (13) the scaling dimensions $\Delta_{1}^{ \pm}$in Eq. (11) are independent on the remaining system parameters, i.e., the effective coupling $\gamma$ and the bosonic fraction $\alpha$. This a consequence of the $\mathrm{SU}(2)$ invariance of the system in this case. The mode $\epsilon_{1}(k)$ is the spinon mode of the unpolarized system, the CFT describing its low-energy properties is an $[\mathrm{SU}(2)]_{1}$ WessZumino-Witten model.

Additional simplifications arise in the strong coupling limit $\gamma \rightarrow \infty$ (i.e., $Q_{0} \rightarrow 0$ ) where $\zeta_{00}=1, \zeta_{10}=0, \zeta_{01}=\zeta_{11}(0)$

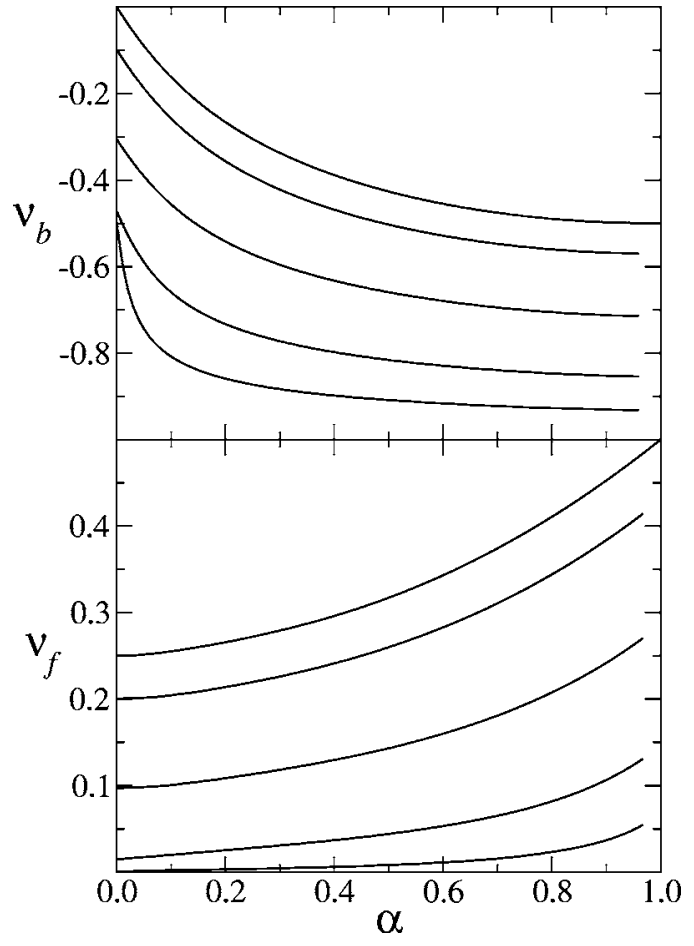

FIG. 1. Exponents characterizing the singularities in the bosonic (upper panel) and fermionic (lower panel) momentum distribution function for the unpolarized gas at $k=0$ and $k_{F}$, respectively, as a function of the bosonic fraction $\alpha$ in the mixture for $\gamma$ $=0.2,1.0,5.0,25.0, \infty$ (bottom to top).

$-1=\alpha$ and $\zeta_{11}(x)$ is given by a scalar integral equation resulting from (14). In Fig. 1 we present results obtained from the numerical solution of these integral equations for the exponents which determine the singularities of the momentum distribution functions for bosons at $k=0$ and fermions at $k=k_{F}$ as a function of the bosonic fraction $\alpha$ for various values of $\gamma$. The exponents $\nu_{b, f}$ at the other wave numbers are always larger than 1 . Note that the system is in a different universality class for $\alpha=0$ or 1 . At $\alpha=0$, all particles are fermionic and the critical exponents are those of the 1D Fermi gas [6]. Here the exponent $\nu_{f}$ for the singularity at the Fermi point varies between 0 and $1 / 8$ as a function of $\gamma$. On the other hand, the limit of $\nu_{b}$ as $\alpha \rightarrow 1$ gives exactly the exponent of the 1D Bose gas with $\delta$ interaction [18].

The spin-polarized gas. Setting $Q_{2}=\infty$ in (3) corresponds to $M_{\downarrow}=0$. This case has been discussed recently in Ref. [7] where some correlation functions have been computed numerically in the strong coupling limit. In this case, the finite size spectrum and the scaling dimensions are determined by two gapless modes. Again, the equations simplify in the strong coupling limit where all exponents can be given as a function of $\alpha$ directly, e.g., $\nu_{b}(0)=\alpha^{2} / 2-\alpha$ and $\nu_{f}\left(k_{F}\right)=\alpha^{2}$ $-\alpha+1 / 2$ for the dominant singularities of the bosonic and fermionic momentum distribution functions, respectively (see Fig. 2 for the $\gamma$ dependence). While the dependence of $\nu_{b}$ on $\alpha$ is similar to the one found in the unpolarized case, the strong coupling behavior of $\nu_{f}$ at a small bosonic fraction is seen to be very different. Note that the singularity at $k_{F}$ $+2 k_{B}$ becomes very pronounced for sufficiently small $\alpha\left(\nu_{f}\right.$ 


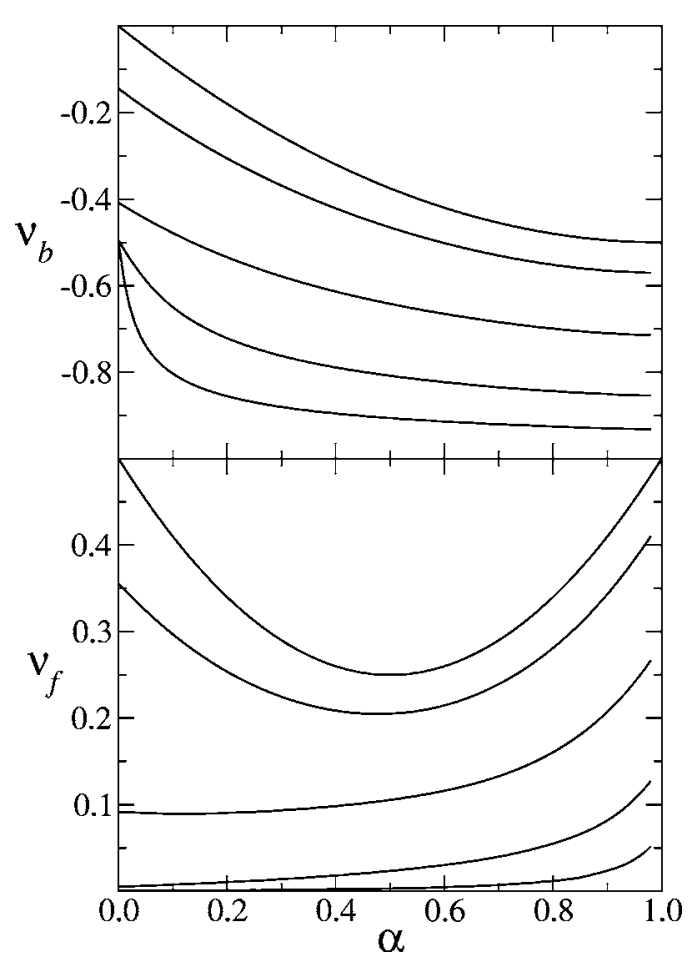

FIG. 2. Same as Fig. 1 for a mixture with polarized fermions.

$=\alpha^{2}+\alpha+1 / 2$ at strong coupling). This feature of the fermionic distribution function is a direct signature of the interaction and should be observable in experiments.

In summary we have used predictions from CFT on the finite size scaling of the low-energy spectrum to study the critical properties of a 1D Bose-Fermi mixture. Generically_not limited to the integrable model (1) considered in this Rapid Communication-the low-energy effective theory of such systems and therefore the asymptotic behavior of its correlation functions is determined by three linearly dispersing modes. Combined with the exact solution of (1) this approach allows to relate the critical exponents directly to the parameters in the microscopic Hamiltonian, i.e., the coupling strength, the fraction of bosons and polarization of the fermions. For two special cases we have studied this relation for the momentum distribution functions and obtained simplified expressions valid in the strong coupling limit. These quantitative predictions indicate how to tune the parameters of a given system for enhanced signatures of interaction which can be detected in experiments such as [1].

Our approach opens new possibilities to investigate the phase diagram of the $1 \mathrm{D}$ mixture by identifying the order parameter with the slowest long-distance decay of its correlation functions (smallest exponent) $[10,11]$. Within the integrable model (1) there is no instability leading to a phase transition $[12,13]$. We emphasize, however, that our expression (11) for the exponents is valid in more general systems (with different coupling constants or longer-ranged interaction) and therefore allows for the study of the phase diagram based, e.g., on numerical data on the spectrum of finite systems.

This work has been supported by the Deutsche Forschungsgemeinschaft.
[1] B. Paredes et al., Nature (London) 429, 277 (2004); S. Richard et al., Phys. Rev. Lett. 91, 010405 (2003).

[2] E. Altman, E. Demler, and M. D. Lukin, Phys. Rev. A 70, 013603 (2004); L. Mathey, E. Altman, and A. Vishwanath, e-print cond-mat/0507108.

[3] A. O. Gogolin, A. A. Nersesyan, and A. Tsvelik, Bosonization and Strongly Correlated Systems (Cambridge University Press, Cambridge, 1998).

[4] E. H. Lieb and W. Liniger, Phys. Rev. 130, 1605 (1963); E. H. Lieb, ibid. 130, 1616 (1963).

[5] K. Mølmer, Phys. Rev. Lett. 80, 1804 (1998); M. Lewenstein et al., ibid. 92, 050401 (2004).

[6] F. H. L. Essler et al., The One-Dimensional Hubbard Model (Cambridge University Press, Cambridge, 2005).

[7] A. Imambekov and E. Demler, e-print cond-mat/0505632.

[8] V. V. Cheianov, H. Smith, and M. B. Zvonarev, Phys. Rev. A 71, 033610 (2005).
[9] M. Ogata and H. Shiba, Phys. Rev. B 41, 2326 (1990).

[10] M. A. Cazalilla and A. F. Ho, Phys. Rev. Lett. 91, 150403 (2003).

[11] L. Mathey et al., Phys. Rev. Lett. 93, 120404 (2004).

[12] C. K. Lai and C. N. Yang, Phys. Rev. A 3, 393 (1971).

[13] M. T. Batchelor et al., e-print cond-mat/0506478.

[14] H. Frahm and V. E. Korepin, Phys. Rev. B 42, 10553 (1990); 43, 5653 (1991).

[15] N. Kawakami and S.-K. Yang, J. Phys.: Condens. Matter 3, 5983 (1991).

[16] A. G. Izergin, V. E. Korepin, and N. Yu. Reshetikhin, J. Phys. A 22, 2615 (1989); F. Woynarovich, ibid. 22, 4243 (1989); H. Frahm and N.-C. Yu, ibid. 23, 2115 (1990).

[17] A. A. Belavin, A. M. Polyakov, and A. B. Zamolodchikov, Nucl. Phys. B 241, 333 (1984).

[18] F. D. M. Haldane, Phys. Rev. Lett. 47, 1840 (1981); M. A. Cazalilla, J. Phys. B 37, S1 (2004). 